\title{
Wavelet Neural Network Application for Kinematic Solution of 2-(6UPS) Hybrid Manipulator
}

\author{
Arash Rahmani, Ahamd Ghanbari and Mehran Mahboubkhah \\ Department of Mechanical Engineering, University of Tabriz,Tabriz, Iran; ara_rahmani@yahoo.com, \\ ag3313656@gmail.com,mahboobkhah@tabrizu.ac.ir
}

\begin{abstract}
This contribution addresses forward kinematic solution of modular hybrid manipulator which includes two same Stewart mechanism in serial form known as 2-(6UPS) manipulator. First, using geometrical and vectorial analysis, mathematical model of kinematic analysis for 2-(6UPS) is extracted. Mapping the length of pods to location (orientation and position) of moving mid and upper platforms (forward kinematic) in this specific mechanism is difficult to solve, as it is related to complicated configuration of 2-(6UPS) and highly nonlinear characteristic of extracted mathematical model. Therefore, we propose artificial neural network (ANN) based wavelet analysis to resolve forward kinematics of 2-(6UPS). Also, we used wavelet neural network (WNN) to approximate specific trajectory in smooth circle and semi-cardioid paths for mid and upper platforms movement respectively. Comparison between the results of proposed network and closed form solution (CFS) of kinematics for 2-(6UPS) shows proper performance of proposed network in less than $1 \%$ error.
\end{abstract}

Keywords: 2-(6UPS) Manipulators, Forward Kinematics Analysis, Nonlinear Multivariable System, Stewart Mechanism, WNN

\section{Introduction}

A hybrid manipulation system is a sequence of parallel mechanisms which can overcome the limited workspace of parallel mechanism and can provide feature of both serial and parallel mechanism. They achieve high stiffness and high force-to-weight ratio. The hybrid serial-parallel robotic manipulator has attracted the attention of many researchers and it also has growing applications to robotics, machine tools, positioning systems, measurement devices, and so on. It has been proved great potential and advantage both closed-loop and opened-loop manipulator over the traditional manipulator. Many different types of hybrid robots have been investigated ${ }^{1-5}$. The characteristics of 6 DOF parallel-serial hybrid manipulators which features a 3 DOF in series actuated module mounted on the moving plate of another 3 DOF in parallel actuated manipulator with prismatic actuators is studied by Huang et $\mathrm{al}^{6}$. Romdhane ${ }^{7}$ investigated the hybrid manipulator which made of a base and two platforms in series and the motion of the mid platform is restricted only to three translations and the second platform rotates spherically with respect to the mid platform using joint connected the mid platform and top platform. Tanio ${ }^{8}$ presented a hybrid (parallel serial) manipulator consisting of two serially connected parallel mechanisms and overall 6DOF and gave its closed-form solution for forward and inverse position problems. The kinematics of hybrid type manipulation system with $6 \mathrm{DOF}$, which consist of a 3-DOF planar parallel platform and a 3-DOF serial robot arm, is discussed by Yang et $\mathrm{al}^{9}$. Huang et $\mathrm{al}^{10}$ studied a 3-DOF parallel robot which it is bottommost of a novel 5-DOF hybrid mechanism. They designed mechanism conceptually and investigated dimensional synthesis of it. LiangZhi et al. ${ }^{11}$ studied a hybrid 5DOF manipulator based on the novel 3-RPS inactuated parallel manipulator. In their design a $2 \mathrm{DOF}$ serial working table is placed over the mobile platform. To analysis hybrid manipulators as a whole structure, Campos et $\mathrm{al}^{12}$ proposed a new method to construct kinematic chains based on assure groups. Gallardo et al. ${ }^{13,14}$ applied screw method and principle of virtual work to analysis kinematics and dynamic of

*Author for correspondence 
a new hybrid mechanism known as 2-(3-RPS). Also, kinematic analysis of a novel specific hyper-redundant mechanism built with a variable number of serially connected identical mechanical modules with autonomous motions is addressed by Gallardo et al. ${ }^{15}$. A new 6DOF hybrid mechanism as 3RPS-3SPR is investigated by $\mathrm{Hu}$ et al. ${ }^{16}$. They studied workspace analysis and inverse kinematics by jacobian methods and active forces by principle of virtual work. Li et al. ${ }^{17}$ used a hybrid manipulator as a multi-dimensional vibration isolator based on the parallel mechanism. The scheme design, inverse kinematics, workspace and dexterity are carried out in their paper. Chen et al. ${ }^{18}$ proposed a multi-objective genetic algorithm trajectory planner for a PKM, based on the dynamics approach. Ghanbari et al. ${ }^{19}$ studied forward kinematic analysis of a new hybrid mechanism with two Stewart module by multi-layer perceptron (MLP) and radial bias function (RBF) neural network.

In this contribution, in order to increase the size of workspace of parallel manipulators, a new hybrid manipulator known as 2-(6UPS) is introduced which includes two same Stewart platforms in serial form. Then, mathematical model of kinematic analysis for 2-(6UPS) is extracted using principle of geometrical and vectorial analysis ${ }^{20,21}$. It is well-known that because of nonlinear geometric relationship between kinematic variables, the solution of forward kinematics of this specific mechanism is too difficult. Therefore, a novel artificial neural network based on wavelet analysis is proposed to solve forward kinematics of 2-(6UPS). Also, we applied WNN to approximate specific trajectory in smooth circle and semi-cardioid paths for mid and upper platforms movement respectively and accurate results are obtained.

\section{Description of the Hybrid Robot}

The mechanism under investigation in this paper consists of two same modules (base and upper modules) that each module is Stewart Platform mechanism with 6 DOFs. In this hybrid mechanism, we have three platforms and twelve pods. Base platform is stationary and connected to middle platform via 6 extensible pods. Also, middle platform is connected to upper platform (as an end effecter) via 6 extensible pods. Each pod connects to the platform at its connection point through a spherical joint, and to the base at its connection point through universal joint. Each pod consists of two parts: the upper part and the lower part, which connect to each other through prismatic joint. Therefore, it is referred to as the 2-(6UPS) mechanism. This manipulator is actuated by motors located on the prismatic joints. Figure 1 shows the design of the mentioned hybrid robot and structure of middle and upper plates. Cartesian coordinate frame is attached to each plate with $\mathrm{z}$ axis normal to the plate.

\section{Forward and Inverse Kinematics Solution}

Mechanism kinematics deals with the study of the mechanism motion as constrained by the geometry of the links. Typically, the study of mechanism kinematics is divided into two parts: inverse kinematics and direct kinematics. About mentioned hybrid robot, the inverse kinematics problem involves mapping a known pose (position and orientation) of the moving platforms of the mechanism to a length of each module's pods. The direct kinematics problem involves the mapping from a known length of each module's pods to a pose of the moving platforms. In this section the inverse and forward kinematics problems of proposed mechanism are described in closed form.
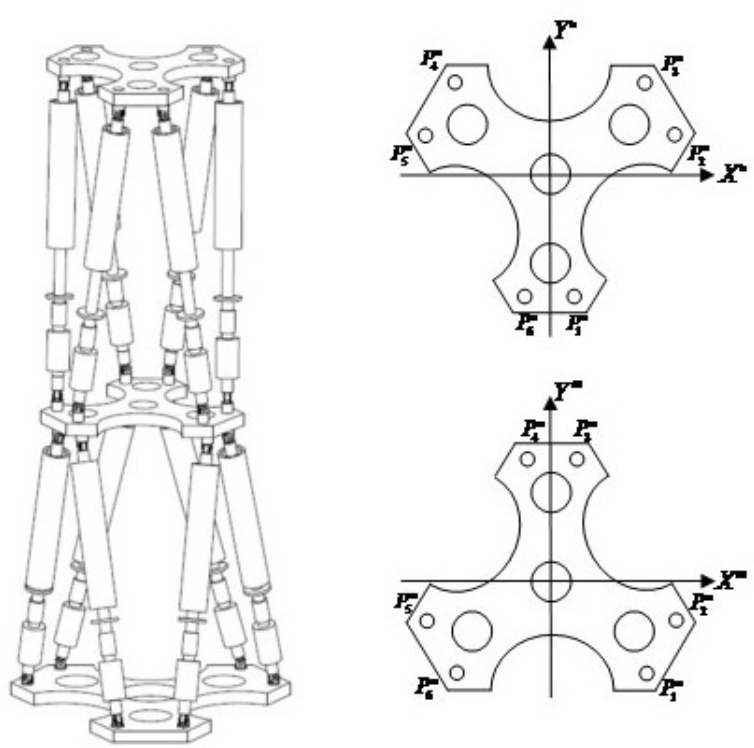

Figure 1. Configuration of 2-(6UPS) hybrid manipulator and plates. 
Figure 2 shows the vectorial representation of the $i^{\text {th }}$ pod at each module. According to Figure 2, the middle and upper moving platforms frame are shown by $\left\{\mathbf{O}^{m}\right\}$ and $\left\{\mathbf{O}^{u}\right\}$ respectively and base frame with $\left\{\mathbf{O}^{b}\right\}$.

Also, $\left(x^{m}, y^{m}, z^{m}, \theta_{x}^{m}, \theta_{y}^{m}, \theta_{z}^{m}\right)$ and $\left(x^{u}, y^{u}, z^{u}, \theta_{x}^{u}, \theta_{y}^{u}, \theta_{z}^{u}\right)$ present the location (position and orientation) of the middle and upper moving platform respectively. Now, the inverse kinematics of each module is obtained at first, and then forward kinematics is considered. Inverse Kinematic problem of the platforms involves determination of the linear position, of six Pods for each module through considering a specified position, of the middle and upper moving platforms centre. For each module, homogeneous transformation matrices from frame $\left\{\mathbf{O}^{u}\right\}$ to frame $\left\{\mathbf{O}^{m}\right\}$ and frame $\left\{\mathbf{O}^{m}\right\}$ to frame $\left\{\mathbf{O}^{b}\right\}$ are $\mathbf{H}_{O^{u}, O^{m}}$ and $\mathbf{H}_{O^{m}, O^{b}}$ respectively, and are described as:

$\begin{aligned} \mathbf{H}_{O^{u}, O^{m}} & =\left[\begin{array}{cccc}R_{1}^{u} & R_{4}^{u} & R_{7}^{u} & x^{u} \\ R_{2}^{u} & R_{5}^{u} & R_{8}^{u} & y^{y} \\ R_{3}^{u} & R_{6}^{u} & R_{9}^{u} & z^{u} \\ 0 & 0 & 0 & 1\end{array}\right] \\ & =\left[\begin{array}{cccc}C \theta_{z}^{u} C \theta_{y}^{u} & -S \theta_{z}^{u} C \theta_{x}^{u}+C \theta_{z}^{u} S \theta_{y}^{u} S \theta_{x}^{u} & S \theta_{z}^{u} S \theta_{x}^{u}+C \theta_{z}^{u} S \theta_{y}^{u} C \theta_{x}^{u} & x^{u} \\ S \theta_{z}^{u} C \theta_{y}^{u} & C \theta_{z}^{u} C \theta_{x}^{u}+S \theta_{z}^{u} S \theta_{y}^{u} S \theta_{x}^{u} & -C \theta_{z}^{u} S \theta_{x}^{u}+S \theta_{z}^{u} S \theta_{y}^{u} C \theta_{x}^{u} & y^{y} \\ -S \theta_{y}^{u} & C \theta_{y}^{u} S \theta_{x}^{u} & C \theta_{y}^{u} C \theta_{x}^{u} & z^{u} \\ 0 & 0 & 0 & 1\end{array}\right] .\end{aligned}$

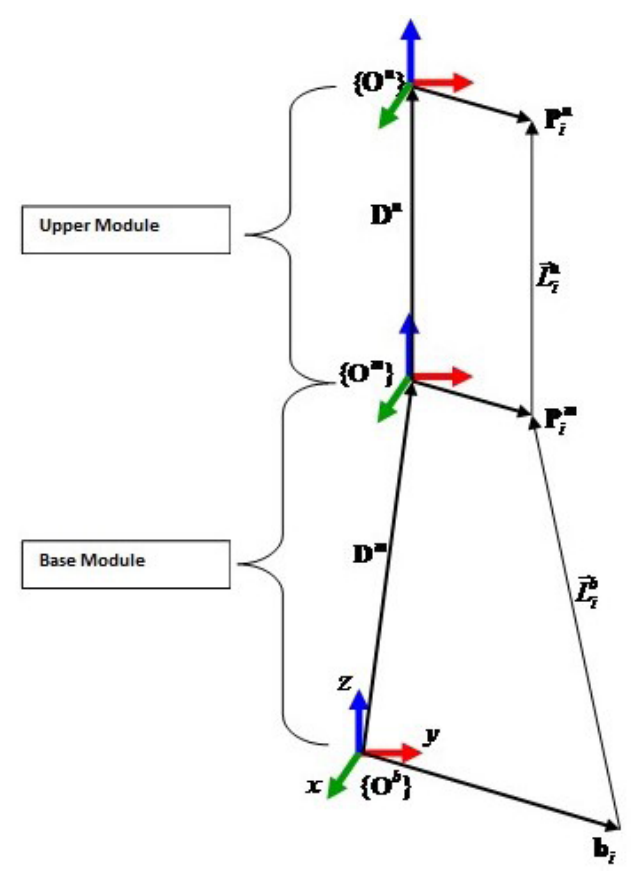

Figure 2. Vectorial representation of the $i^{\text {th }}$ pod at each module.

$$
\begin{aligned}
\mathbf{H}_{O^{m}, O^{b}} & =\left[\begin{array}{cccc}
R_{1}^{m} & R_{4}^{m} & R_{7}^{u} & x^{m} \\
R_{2}^{m} & R_{5}^{m} & R_{z}^{u} & y^{m} \\
R_{3}^{m} & R_{6}^{m} & R_{9}^{u} & z^{m} \\
0 & 0 & 0 & 1
\end{array}\right] \\
& =\left[\begin{array}{cccc}
C \theta_{z}^{m} C \theta_{y}^{m} & -S \theta_{z}^{m} C \theta_{x}^{m}+C \theta_{z}^{m} S \theta_{y}^{m} S \theta_{x}^{m} & S \theta_{z}^{m} S \theta_{x}^{m}+C \theta_{z}^{m} S \theta_{y}^{m} C \theta_{x}^{m} & x^{m} \\
S \theta_{z}^{m} C \theta_{y}^{m} & C \theta_{z}^{m} C \theta_{x}^{m}+S \theta_{z}^{m} S \theta_{y}^{m} S \theta_{x}^{m} & -C \theta_{z}^{m} S \theta_{x}^{m}+S \theta_{z}^{m} S \theta_{y}^{m} C \theta_{x}^{m} & y^{m} \\
-S \theta_{y}^{m} & C \theta_{y}^{m} S \theta_{x}^{m} & C \theta_{y}^{m} C \theta_{x}^{m} & z^{m} \\
0 & 0 & 0 & 1
\end{array}\right] .
\end{aligned}
$$

Where $C \theta=\cos (\theta), S \theta=\sin (\theta)$.

Beside, ${ }^{O^{m}} \mathbf{L}_{i}^{u}$ and ${ }^{O^{b}} \mathbf{L}_{i}^{u}$ are the vector of length of the $i^{\text {th }}$ pod in upper module relative to $\mathbf{O}^{m}$ and $\mathbf{O}^{b}$ frame respectively. And, ${ }^{O^{b}} \mathbf{L}_{i}^{b}$ is the vector of length of the $i^{t h}$ pod in base module relative to $\mathbf{O}^{b}$ frame. Vector of length of the $i^{\text {th }}$ pod without considering modules and frames, is a $1 \times 4$ vector which is described as:

$$
\mathrm{L}_{i}=\left(\mathrm{L}_{\mathrm{ix}}, \mathrm{L}_{\mathrm{iy}}, \mathrm{L}_{\mathrm{iz}}, 1\right)
$$

Also, $\mathbf{b}_{i}, \mathbf{P}_{i}^{m}, \mathbf{P}_{i}^{u}$ are the six vertices of the base, middle and upper plates relative to $\mathbf{O}^{b}, \mathbf{O}^{m}$ and $\mathbf{O}^{u}$ frame respectively:

$$
\begin{array}{ll}
\mathbf{b}_{i}=\left(b_{i x}, b_{i y}, 0,1\right) & i=1,2, \ldots, 6 . \\
\mathbf{P}_{i}^{m}=\left(p_{i x}^{m}, p_{i y}^{m}, 0,1\right) & i=1,2, \ldots, 6 . \\
\mathbf{P}_{i}^{u}=\left(p_{i x}^{u}, p_{i y}^{u}, 0,1\right) & i=1,2, \ldots, 6 .
\end{array}
$$

Now, according to transformation of a vector from one frame to another, we can determine ${ }^{O^{b}} \mathbf{L}_{i}^{b},{ }^{O^{m}} \mathbf{L}_{i}^{u}$ and ${ }^{O^{b}} \mathbf{L}_{i}^{u}$ as:

$$
\begin{gathered}
O^{b} \mathbf{L}_{i}^{b}=\left(\mathbf{H}_{O^{m}, O^{b}} \mathbf{P}_{i}^{m}-\mathbf{b}_{i}\right) . \\
O^{m} \mathbf{L}_{i}^{u}=\left(\mathbf{H}_{O^{u}, O^{m}} \mathbf{P}_{i}^{u}-\mathbf{P}_{i}^{m}\right) . \\
{ }^{b} \mathbf{L}_{i}^{u}=\mathbf{H}_{O^{m}, O^{b}}{ }^{O^{m}} \mathbf{L}_{i}^{u}=\mathbf{H}_{O^{m}, O^{b}}\left(\mathbf{H}_{O^{u}, O^{m}} \mathbf{P}_{i}^{u}-\mathbf{P}_{i}^{m}\right) .
\end{gathered}
$$

Equations (7) and (9), define the length of pods of each module relative to base in a closed form. Mapping the pose of middle and upper plates to the length of pods is easy and straightforward. But, because of highly nonlinear characteristic of these equations, the mapping from a known length of each module's pods to a pose of the moving platforms is so difficult and too much 
complicated. Therefore, wavelet based neural network (wave-net) is applied to solve forward kinematics of this mechanism.

\section{Wavelet Neural Network}

\subsection{Structure of Network}

The wavelet neural network (WNN) is the model based on wavelet transformation and artificial neural network ${ }^{22}$. Due to wavelet transform has the good localization characteristics in time domain and neural networks has the good ability to approximate complicated maps, WNN incorporate the good learning ability and the good property of localization, which have been successfully applied in function approximation and pattern classification. Proposed neural network is established by conjunction of neurons in the forward direction.

Because the approximation class is nonlinear in the adjustable parameters, the training procedure of the neural networks may become trapped in some local minimum depending on the initialization. To overcome this problem, the wavelet networks have been proposed as an alternative to neural networks, which follow the availability of rates of convergence for approximation by wavelet based networks.

In this section a feed forward single hidden layer network is introduced. For a (back propagation) BP neural network with only one hidden layer of neurons, using basis wavelets as its activate functions of hidden layer, we get a multi-input and multi-output wavelet neural networks in Figure 3.

This WNN has $\mathrm{m}, \mathrm{p}, \mathrm{n}$ nodes in the input layer, hidden layer and output layer respectively. And the activate function of the $j^{\text {th }}$ node in the hidden layer is ${ }^{23}$ :

$$
\psi_{a_{j}, b_{j}}(t)=\frac{1}{\sqrt{a_{j}}} \psi\left(\frac{t-b_{j}}{a_{j}}\right) \quad j=1,2, \ldots, p .
$$

Where, $\Psi(t)$ is the mother wavelet function which is localized both in time and frequency and could be chosen as different function according to the feature of the problem. Figure 4 shows four types of most applicable wavelet functions. In this paper we use Mexican Hat wavelet. This wavelet is derived from a function, which is proportional to the second derivative function of the Gaussian probability density function. It is non-orthogonal, with infinite

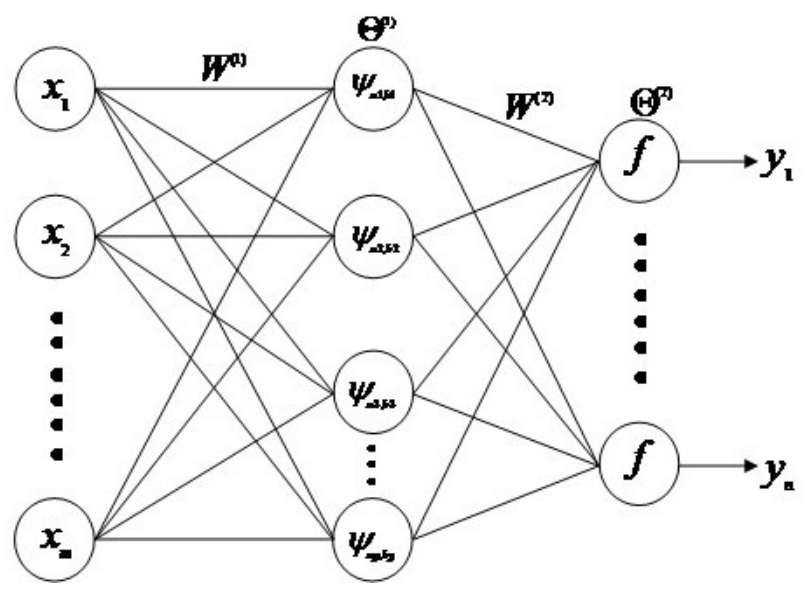

Figure 3. Structure of WNN.

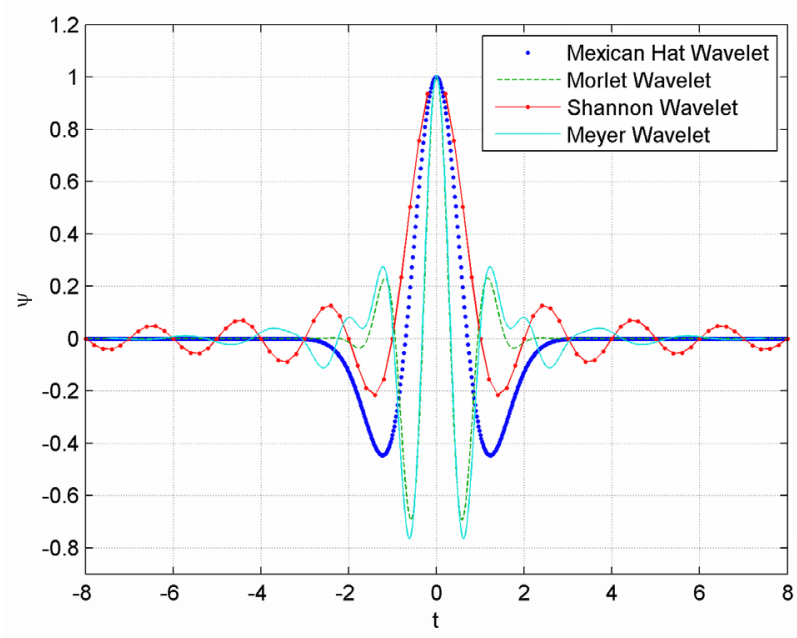

Figure 4. Wavelet Functions.

support and has maximum energy around origin with the narrow band. The expression for Mexican Hat wavelet is given by Eq. (11). In this paper, $f(t)$ is chosen as sigmoid function.

$$
\psi(t)=\left(1-2 t^{2}\right) \cdot \exp \left(-t^{2}\right)
$$

The wavelet neural network parameters, $\left(W^{(1)}, W^{(2)}, \Theta^{(1)}, \Theta^{(2)}, a_{1}, \ldots, a_{p}, b_{1}, \ldots, b_{p}\right)$ in Figure 3, should be adjusted through training.

\subsection{Training of Network}

Back propagation method is the most frequently used technique for training a feed forward network. It involves 
two passes across the network, forward and backward pass. The forward pass supply the network's output performance and the backward implicates effluence the error initially found in the output node back across the network to specify errors for each node which contributed to the initial error. When all the errors are specified, for minimizing these errors the weights are changed. Since the WNN in Figure 3 is derived from a feed forward neural network, we use back propagation method to train this network. For the WNN in Figure 3, when the input vector is $\mathbf{X}=\left(x_{1}, x_{2}, \ldots, x_{m}\right)$, we get the output of the $j^{\text {th }}$ node in hidden layer:

$$
\begin{aligned}
\psi_{a_{j}, b_{j}}\left(\sum_{k=1}^{m} w_{j k}^{(1)} x_{k}-\theta_{j}^{(1)}\right) & =\psi_{a_{j}, b_{j}}\left(F_{j}^{(1)}\right) \\
& =\frac{1}{\sqrt{a_{j}}} \psi\left(\frac{F_{j}^{(1)}-b_{j}}{a_{j}}\right) .
\end{aligned}
$$

Where,

$$
F_{j}^{(1)}=\sum_{k=1}^{m}\left(w_{j k}^{(1)} x_{k}-\theta_{j}^{(1)}\right)
$$

the output of the $i^{\text {th }}$ node of output layer is:

$$
y_{i}=f\left(\sum_{j=1}^{p} w_{i j}^{(2)} \psi_{a j, b j}\left(F_{j}^{(1)}\right)-\theta_{i}^{(2)}\right)=f\left(F_{i}^{(2)}\right) .
$$

Where,

$$
F_{i}^{(2)}=\sum_{j=1}^{p}\left(w_{i j}^{(2)} \psi_{a j, b j}\left(F_{j}^{(1)}\right)-\theta_{i}^{(2)}\right)
$$

From equation (19) we get the output vector of the WNN: $\mathbf{Y}=\left(y_{1}, y_{2}, \ldots, y_{n}\right)$. Suppose we have $Q$ training samples. For each sample $q$, the desired output vector is, $\overline{\mathbf{Y}}_{q}=\left(\bar{y}_{q 1}, \bar{y}_{q 2}, \ldots, \bar{y}_{q n}\right)$, the output vector of the WNN is $\mathbf{Y}_{q}=\left(y_{q 1}, y_{q 2}, \ldots, y_{q n}\right)$. With these $\mathrm{Q}$ training samples, we train the WNN through batch learning process. Then the main goal for proposed network is providing minimum total error $\mathrm{E}$ for each output node $i$ over all training samples ${ }^{24}$ :

$$
E=\frac{1}{2} \sum_{q=1}^{Q} \sum_{i=1}^{n}\left(\bar{Y}_{q i}-Y_{q i}\right)^{2} .
$$

By the iterative gradient descent method, the parameters of the wavelet neural network can be formulated by:

$$
\begin{gathered}
\left.w_{i j}^{(2)}(t+1)=(1+\beta) w_{i j}^{(2)}(t)-\beta w_{i j}^{(2)}(t-1)-\lambda \frac{\partial E}{\partial w_{i j}^{(2)}}\right) . \\
w_{j k}^{(1)}(t+1)=(1+\beta) w_{j k}^{(1)}(t)-\beta w_{j k}^{(1)}(t-1)-\lambda \frac{\partial E}{\partial w_{j k}^{(1)}} . \\
\theta_{i}^{(2)}(t+1)=(1+\beta) \theta_{i}^{(2)}(t)-\beta \theta_{i}^{(2)}(t-1)-\lambda \frac{\partial E}{\partial \theta_{i}^{(2)}} . \\
\theta_{j}^{(1)}(t+1)=(1+\beta) \theta_{j}^{(1)}(t)-\beta \theta_{j}^{(1)}(t-1)-\lambda \frac{\partial E}{\partial \theta_{j}^{(1)}} . \\
a_{j}(t+1)=(1+\beta) a_{j}(t)-\beta a_{j}(t-1)-\lambda \frac{\partial E}{\partial a_{j}} . \\
b_{j}(t+1)=(1+\beta) b_{j}(t)-\beta b_{j}(t-1)-\lambda \frac{\partial E}{\partial b_{j}} .
\end{gathered}
$$

Where $t$ is the iteration index of learning and $\lambda$ is the learning rate. To improve the rate of learning, we modify the original learning rule with the momentum factor $\beta(0<\beta<1)$ to the weights ${ }^{25}$. The partial derivatives of the error $\mathrm{E}$ respect to each parameter can be calculated easily.

\section{WNN Solution for Kinematics of Robot}

In order to model forward Kinematics of hybrid robot with wave-net, according to structure of robot. We have modeled the base module and the upper, respectively. The input data of the network are the length of pods for each module. At first, using the length of pods of the base module, $\mathbf{L}^{b}=\left(l_{1}^{b}, l_{2}^{b}, l_{3}^{b}, l_{4}^{b}, l_{5}^{b}, l_{6}^{b}\right)$, we define the location (position and orientation) for mid platform, $P^{m}=\left(x^{m}, y^{m}, z^{m}, a^{m}, \beta^{m}, \gamma^{m}\right)$. Next, using the length of pods for upper module, $\mathbf{L}^{u}=\left(l_{1}^{u}, l_{2}^{u}, l_{3}^{u}, l_{4}^{u}, l_{5}^{u}, l_{6}^{u}\right)$, and the position and orientation of the middle plate, we calculated the location (position and orientation) for upper platform as an end effecter location, $P^{u}=\left(x^{u}, y^{u}, z^{u}, a^{u}, \beta^{u}, \gamma^{u}\right)$. So, there will be two different and separate networks. 
For approximating the kinematics of hybrid manipulator, the algorithm of WNN is summarized as follows:

Step 1: Set the initial values of networks parameters, $\left(W^{(1)}, W^{(2)}, \Theta^{(1)}, \Theta^{(2)}, a_{1}, \ldots, a_{p}, b_{1}, \ldots, b_{p}\right) \quad$ learning rate $\lambda$ and momentum factor $\beta$.

Step 2: Input the training data and the desired output values. Give input vectors $\mathbf{X}=\left(x_{1}, x_{2}, \ldots, x_{m}\right)$ where it is thelength of pods of each module and a desired output vector $\overline{\mathbf{Y}}_{q}=\left(\bar{y}_{q 1}, \bar{y}_{q 2}, \ldots, \bar{y}_{q n}\right)$, the theoretical values acquired from inverse kinematic solution of Eqs. (7) and (9).

Step 3: For each input datum, calculate the output of the wavelet neural network by Eq. (14).

Step 4: Adjust the networks parameters $\left(W^{(1)}, W^{(2)}\right.$, $\left.\Theta^{(1)}, \Theta^{(2)}, a_{1}, \ldots, a_{p}, b_{1}, \ldots, b_{p}\right) \quad$ using gradient descent algorithm by Eqs. (17) to (22).

Step 5: The error function $\mathrm{E}$ is calculated by Eq. (16). If the error is less than the desired bound, the networks parameters are obtained and the learning process is terminated, else go to step 2.

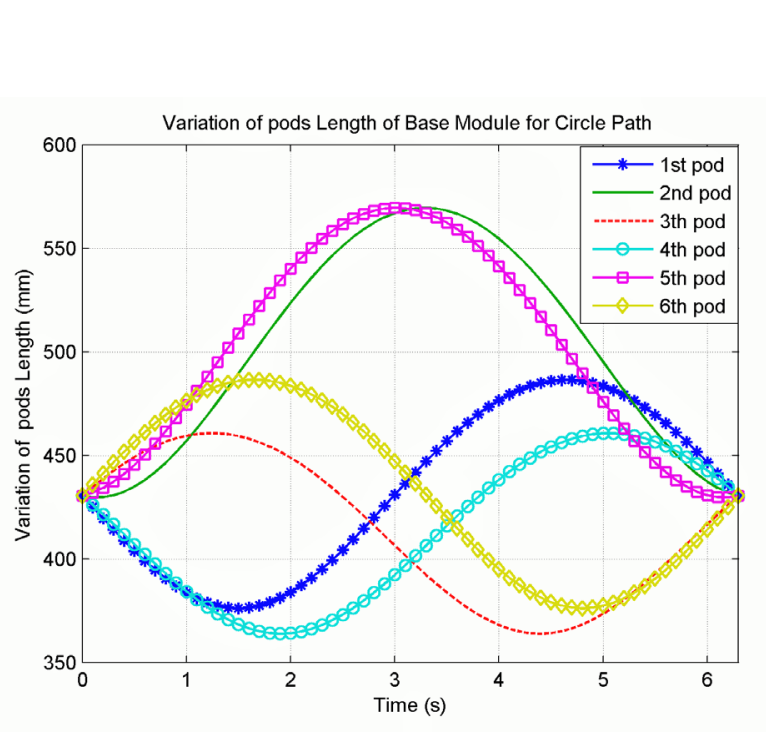

Figure 5. Variation of length of pods. (a) Base module for circle path with Orientation $\left(\left[0^{\circ}, 0^{\circ}, 0^{\circ}\right]\right)$ (b) Upper module for semi-cardioid path with Orientation $\left(5^{*}\left[0^{\circ},-2 \sin (t)^{\circ}, \sin (t / 2)^{\circ}\right]\right)$.

\section{Results}

In this section, we present the results of the proposed WNN on approximating the kinematic analysis of hybrid robot. The architecture was used for the wavelet network is one input layer with six neurons, one hidden layer with 72 neurons and one output layer with six neurons. The network was trained with a learning rate of 0.17 , a momentum term of 0.1 , and 1,024 learning iterations. The largest error $\mathrm{E}$ or given precision is $1 \%$.

Figures 5 to 8 show the results of the proposed WNN for the specific paths of middle and upper plates of hybrid robot. Results given here are for circle path and semi-cardioid path for center point of middle and upper plate, respectively. Using inverse kinematic analysis for proposed paths of plates, we define motions of each pod of each module (Figure 5). Then, we feed the proposed WNN by the pods motions to get the paths of plates. Figure 6 shows the position paths and Figure 7 shows orientation paths for center point of each plate. Again, we used the outputs of the proposed WNN for inverse kinematic analysis to define the new motions of each pod of each module and compared them with the pods motions which results from CFS. Figure 8 show the results, for variation of length of each pods of base and upper module for circle path for center point of mid plate with $\left(\left[0^{\circ}, 0^{\circ}, 0^{\circ}\right]\right)$

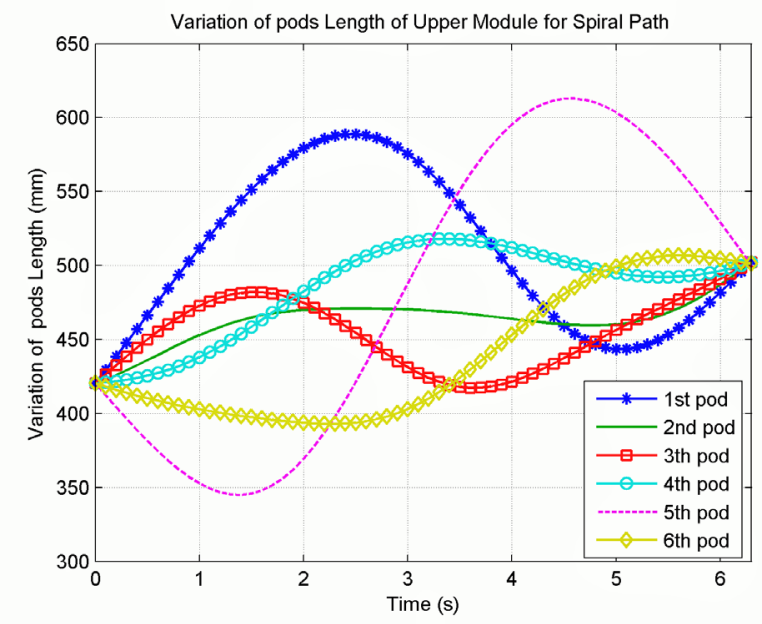
step 2. Vol 7 (12) | December $2014 \mid$ www.indjst.org 

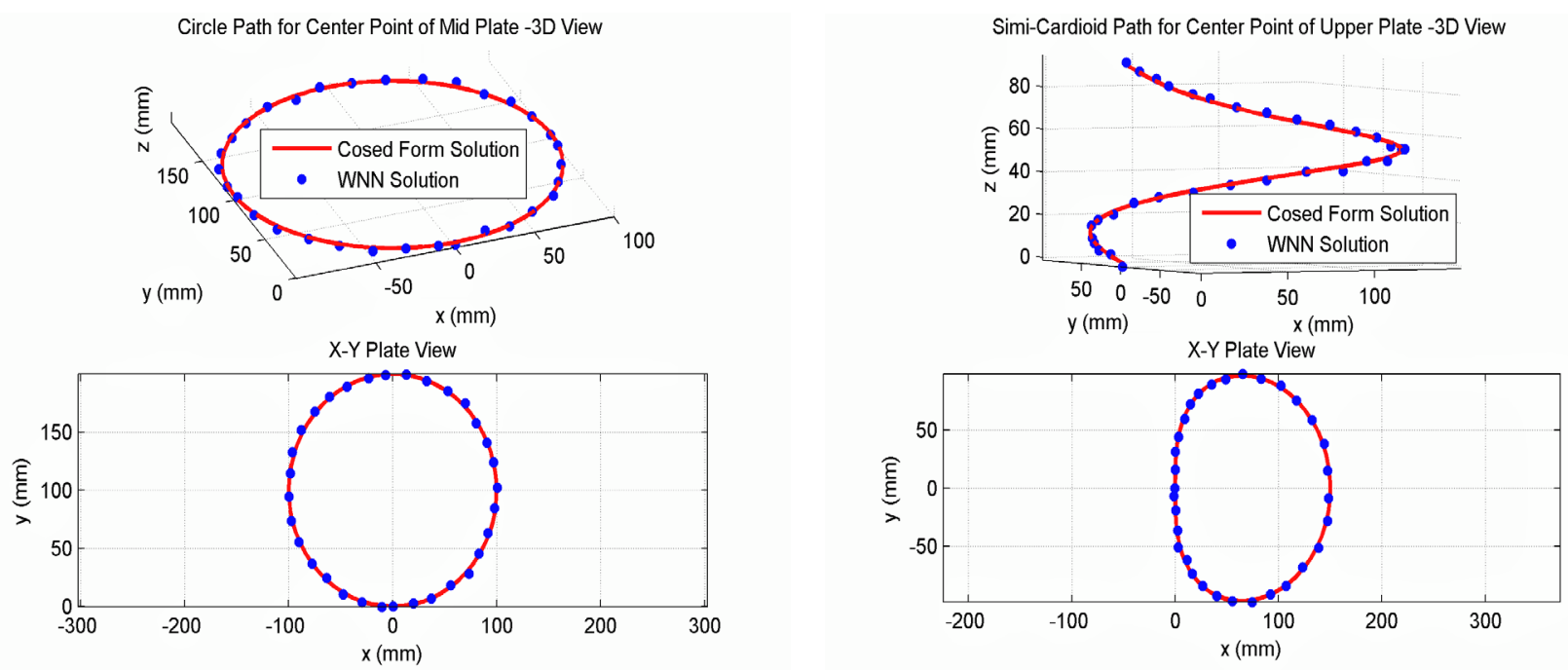

Figure 6. (a) Circle Path Center Point of Mid Plate with Orientation $\left(\left[0^{\circ}, 0^{\circ}, 0^{\circ}\right]\right)$. (b) Semi-Cardioid Path for Center Point of Upper Plate with Orientation $\left(5^{*}\left[0^{\circ},-2 \sin (t)^{\circ}, \sin (t / 2)^{\circ}\right]\right)$.
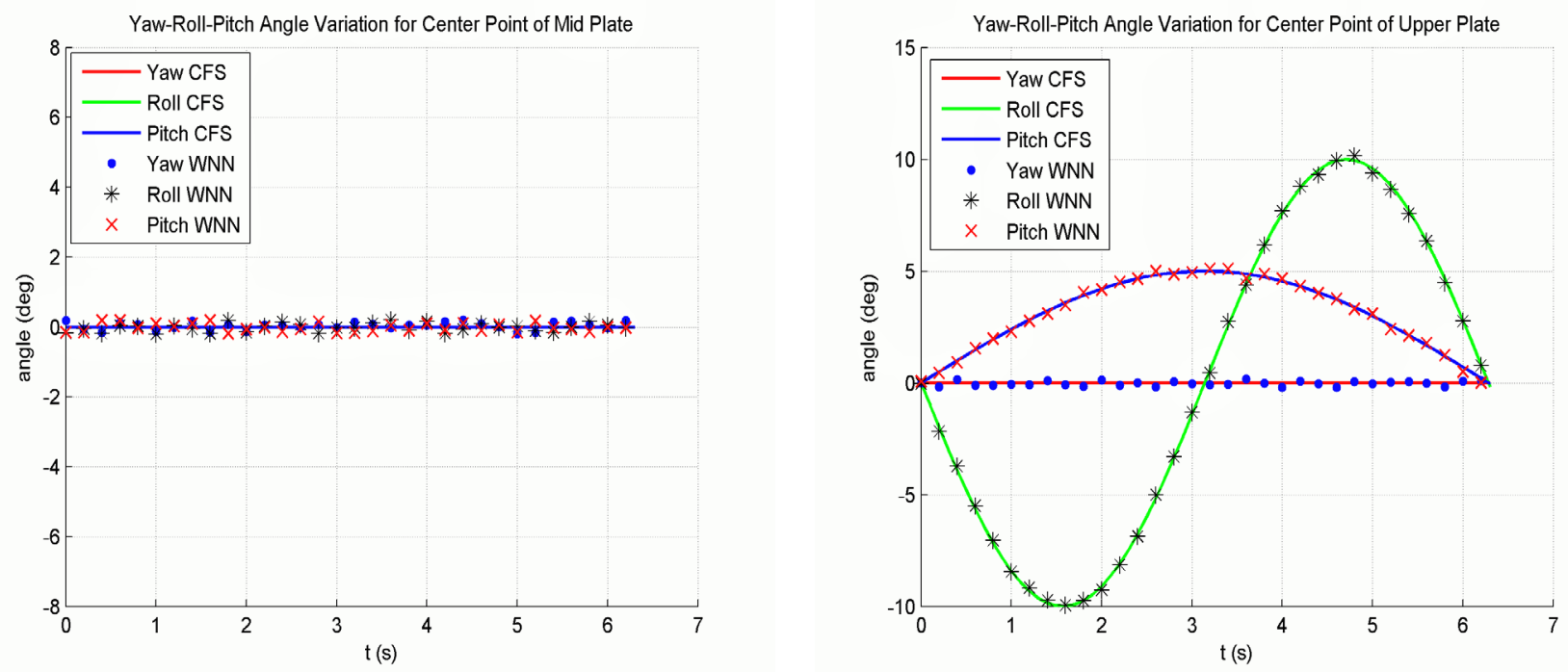

Figure 7. Orientation Path for Center Point (a) Mid Plate (b) Upper Plate.

orientation and semi-cardioid path for center point of upper plate with $\left(5^{*}\left[0^{\circ},-2 \sin (t)^{\circ}, \sin (t / 2)^{\circ}\right]\right)$ orientation.

The results show good agreement between exact solution (CFS) and outputs of proposed WNN. Although, the accumulation of error for kinematic analysis of upper module, causes the error percentage in results of upper plate position and orientation is higher than mid plate. Accumulation of error for kinematic analysis of upper module is derived:

1- Network error

2- Input error (the outputs of WNN for base module with error, are also fixed inputs for WNN for upper plates) 

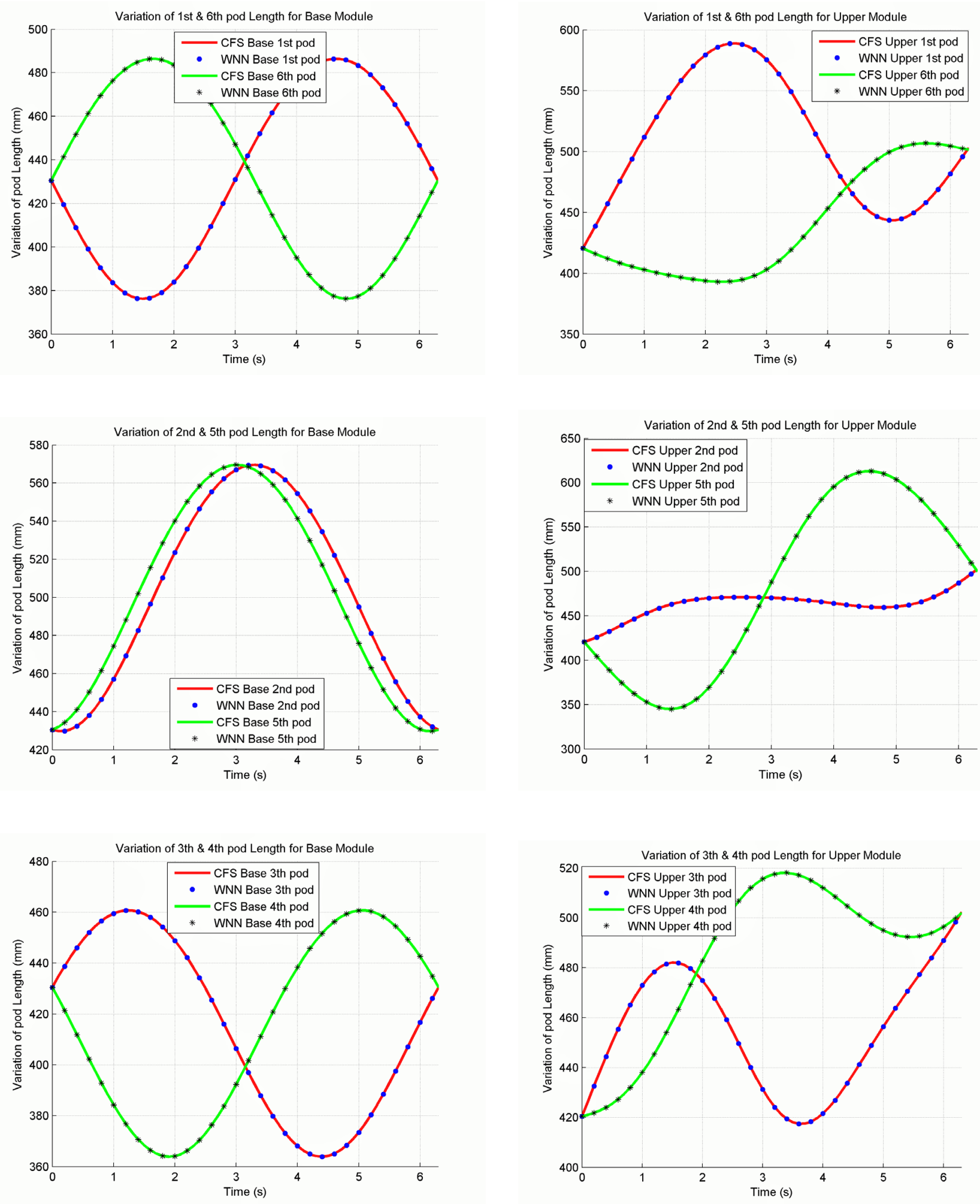

Figure 8. Length Variation of pods of base and upper module for circle and semi-cardioid path respectively with defined orientation change. (a), (c), (e) - for Base Module (b), (d), (f) - for Upper Module. 


\section{Conclusion}

In this paper, a wavelet neural network which can be employed as a useful tool for nonlinear mapping problem has been proposed for solving direct kinematics of hybrid robot. The proposed network can be proved to have the capability of approximating any multivariable systems. Whereas, the kinematics model of hybrid robot has strongly nonlinear characteristic, the network can not only be trained in a short time, but also shows better performance in solving problems. There is good agreement between WNN and CFS. But, because of accumulation of error, the error of results of upper plate is more than the error of the mid plate; although networks training approach shows the maximum error for all cases is less than $1 \%$.

\section{References}

1. Rico J, Gallardo J, Duffy J. Screw theory and higher order kinematic analysis of open serial and closed chains. Mechanism and Machine Theory. 1999; 34(4):559-86.

2. Carbone G, Ceccarelli M. A stiffness analysis for a hybrid parallel-serial manipulator. Robotica. 2004; 22(05):567-76.

3. Carbone G, Ceccarelli M. A serial-parallel robotic architecture for surgical tasks. Robotica. 2005; 23(03):345-54.

4. Zhou B, Xu Y. Robust control of a 3-DOF hybrid robot manipulator. The International Journal of Advanced Manufacturing Technology. 2007; 33(5-6):604-13.

5. Cheng G, Gu W, Ge SR. Kinematic analysis of a 3SPS+1PS parallel hip joint simulator based on Rodrigues parameters. Journal of mechanical science and technology. 2012; 26(10):3299-310.

6. Huang MZ, Ling SH, Sheng Y. A study of velocity kinematics for hybrid manipulators with parallel-series configurations. IEEE International Conference on Robotics and Automation. 1993. IEEE.

7. Romdhane L. Design and analysis of a hybrid serial-parallel manipulator. Mechanism and Machine Theory. 1999; 34(7):1037-55.

8. Tanev TK. Kinematics of a hybrid (parallel-serial) robot manipulator. Mechanism and Machine Theory, 2000; 35(9):1183-96.

9. Yang G, Chen W, Ho EHL. Design and kinematic analysis of a modular hybrid parallel-serial manipulator. 7th International Conference on Control, Automation, Robotics and Vision, ICARCV 2002; 2002; IEEE.

10. Huang T, et al. Conceptual design and dimensional synthesis for a 3-DOF module of the TriVariant-a novel 5-DOF reconfigurable hybrid robot. IEEE Transactions on Robotics. 2005; 21(3):449-56.
11. LiangZhi F, Elatta A, XiaoPing L. Kinematic calibration for a hybrid 5DOF manipulator based on 3-RPS in-actuated parallel manipulator. The International Journal of Advanced Manufacturing Technology. 2005; 25(7-8):730-4.

12. Campos A, Budde C, Hesselbach J. A type synthesis method for hybrid robot structures. Mechanism and Machine Theory. 2008; 43(8):984-95.

13. Gallardo-Alvarado J, et al. Kinematics and dynamics of 2 (3-RPS) manipulators by means of screw theory and the principle of virtual work. Mechanism and Machine Theory. 2008; 43(10):1281-94.

14. Gallardo-Alvarado J, et al. Solving the kinematics and dynamics of a modular spatial hyper-redundant manipulator by means of screw theory. Multibody System Dynamics, 2008; 20(4):307-25.

15. Gallardo J, et al. The kinematics of modular spatial hyperredundant manipulators formed from RPS-type limbs. Robotics and Autonomous Systems. 2011; 59(1):12-21.

16. $\mathrm{Hu} \mathrm{B}$, et al. Analyses of inverse kinematics, statics and workspace of a novel 3RPS-3SPR serial-parallel manipulator. Open Mech Eng J. 2012; 6:65-72.

17. Li B, Zhao W, Deng Z. Modeling and analysis of a multidimensional vibration isolator based on the parallel mechanism. Journal of Manufacturing Systems. 2012; 31(1):50-8.

18. Chen C-T, Pham H-V. Trajectory planning in parallel kinematic manipulators using a constrained multi-objective evolutionary algorithm. Nonlinear Dynamics. 2012; 67(2):1669-81.

19. Ahmad Ghanbari AR. Neural Network Solutions for Forward Kinematics Problem of Hybrid Serial-Parallel Manipulator. World of Sciences Journal. 2013; 1(8):148-58.

20. Qazani MRC, et al. An experimental study on motion error of hexarot parallel manipulator. The International Journal of Advanced Manufacturing Technology. 2014; 72(9-12):1361-76.

21. Qazani MRC, et al. Kinematic analysis and workspace determination of hexarot-anovel6-DOF parallelmanipulator with a rotation-symmetric arm system. Robotica. 1-18.

22. Zhang Q, Benveniste A. Wavelet networks. IEEE Transactions on Neural Networks. 1992; 3(6): 889-98.

23. Banakar A, Azeem MF. Artificial wavelet neuro-fuzzy model based on parallel wavelet network and neural network. Soft Computing. 2008; 12(8):789-808.

24. Huang M, Cui B. A novel learning algorithm for wavelet neuralnetworks. Advancesin NaturalComputation.Springer. 2005; $1-7$.

25. Chen D, Han J. Application of wavelet neural network in signal processing of MEMS accelerometers. Microsystem technologies. 2011; 17(1):1-5. 